\title{
Gas-phase prebiotic chemistry in extraterrestrial environments
}

\author{
Nadia Balucani \\ Dipartimento di Chimica, Università degli Studi di Perugia, Perugia, Italy \\ email: nadia.balucani@unipg.it
}

\begin{abstract}
A variety of molecular species up to complex polyatomic molecules/radicals have been identified in many extraterrestrial gaseous environments, including interstellar clouds, cometary comae and planetary atmospheres. Amongst the identified molecules/radicals, a large percentage are organic in nature and encompass also prebiotic molecules. Different types of microscopic processes are believed to be involved in their formation, including surface processes, ion- and radical- molecule reactions. A thorough characterization of such a complex chemistry relies on a multi-disciplinary approach, where the observations are complemented by accurate chemical modeling. Unfortunately, a literature survey reveals that only a small percentage of the elementary reactions considered in the available models have been characterized in laboratory experiments. In this contribution, a brief overview will be given of recent experimental techniques that have allowed us to reach a better description of neutral-neutral gas-phase reactions, which might be responsible for the formation of simple prebiotic molecules.
\end{abstract}

In the sequence of steps which are believed to have led from elementary particles to the dawn of life, an important one is certainly the formation of simple prebiotic molecules from parent species abundant in the Universe. The combination of $\mathrm{H}, \mathrm{O}, \mathrm{C}, \mathrm{N}, \mathrm{S}$ and other atoms into molecules and their subsequent chemical evolution in extraterrestrial environments are witnessed by the identification of more than one hundred molecules in the interstellar clouds (encompassing also prebiotic molecules such as hydrogen cyanide, glycolaldehyde, formamide) and by the gas-phase chemical evolution of the atmospheres of several solar objects like Titan. The formations of organic molecules/radicals can certainly be regarded as simple processes when compared to other unknown phenomena that have led to the first living organisms. Still, the formation mechanisms of many of the observed gaseous organic molecules/radicals are not well-characterized, while a comprehension of these processes can certainly help to understand the preconditions for the birth of life (Balucani 2009).

Different types of molecular processes are believed to be involved, including radiative association and recombination, surface-induced processes, photon- or particle-induced ionization and ion-molecule reactions, photon- or particle-induced dissociation and radicalmolecule reactions. The concomitance of all these phenomena and the complexity of the considered environments require a modeling approach, where all the relevant molecular processes compatible with the boundary conditions should be included with the appropriate parameters that describe them. As far as neutral-neutral reactions are concerned, provided that they are thermodynamically feasible, the relevant parameters are $(i)$ the kinetic rate constant and $(i i)$ the product branching ratio. Given the low temperature of interstellar clouds and several solar objects, like Titan, the kinetic rate constants should be measured at temperatures as low as $\mathrm{T}=10-100 \mathrm{~K}$.

There are two experimental approaches to cool gases and study their reactions: cryogenic cooling and expansion methods. Cryogenic cooling is limited by the saturation vapour pressure of the reactant gases, while supersonic expansion through a collimating 
axisymmetric, converging-diverging Laval nozzle, leads to the production of a cold and uniform supersonic flow for many gas mixtures. This is the approach used in the CRESU (Cinétique de Réaction en Ecoulement Supersonique Uniforme) technique, which has allowed measuring rate constants down to very low $\mathrm{T}$ (as low as $15 \mathrm{~K}$ ) for many neutralneutral reactions (Smith \& Rowe 2000). From these studies experimental evidence has been obtained that some reactions, including those leading to prebiotic molecules like cyanoacetylene, are very fast, with rate constants in the gas kinetics range at very low $\mathrm{T}$. Also, the CRESU results have pointed out that the extrapolation at very low $\mathrm{T}$ of the Arrhenius dependence of the rate constant outside the range of $\mathrm{T}$ investigated (commonly used in astrochemical modeling) is not warranted.

Elegant as the CRESU studies are, they do not allow determining the nature of the products and their branching ratio. Nevertheless, this piece of information is fundamental to constructing a realistic model, because the products of one reaction are the reactants of a subsequent one. In this respect, a powerful technique is the crossed molecular beam (CMB) method with mass spectrometric (MS) detection. In CMB experiments bimolecular reactions are studied under well-defined conditions. Differently to a bulk experiment, the reacting species are confined in separate beams which cross each other at a specific angle and collision energy. The products are formed at the collision center and then fly undisturbed towards the detector because of the large mean free path achieved by operating at a very low pressure. In this way, the results of well-defined molecular collisions are observed and secondary/wall collisions avoided. The use of MS detection makes the method universal, as any species can be ionized in the ionizer which precedes the mass filter and it is possible to determine the mass and the gross formula of all possible products of a bimolecular reaction. In recent years the CMB-MS method has been successfully applied to the study of reactions leading to prebiotic molecules. For example, several reactions producing nitriles (Balucani et al. 2009, Balucani \& Kaiser 2001, Gu et al. 2009), organosulphur (Leonori et al. 2009) and organophosphorus (Guo et al. 2007) compounds have been characterized.

In conclusion, powerful experimental techniques devoted to the study of neutral-neutral reactions can significantly contribute to reach an unprecedented knowledge of the plausible gas-phase chemistry that has preceded the appearance of life.

\section{Acknowledgements}

Financial support from the Italian MIUR (Ministero Istruzione Università Ricerca) under projects PRIN (2007H9S8SW_004) is acknowledged.

\section{References}

Balucani, N. 2009, Int. J. Mol. Sci. 10, 2304.

Smith, I. W. M. \& Rowe, B. R. 2000, Acc. Chem. Res. 33, 261.

Balucani, N., Bergeat, A., Cartechini, L., Volpi, G. G., \& Casavecchia, P. 2009, J. Phys. Chem. $A$, in press, DOI: $10.1021 /$ jp904302g

Balucani, N. \& Kaiser, R. I. 2001, Acc. Chem. Res. 34, 699.

Gu, X., Kaiser, R. I., Mebel, A. M., Kislov, V. V., Klippenstein, S. J., Harding, L. B., Liang, M. C., \& Yung, Y. L. 2009, ApJ 701, 1797.

Leonori, F., Petrucci, R., Balucani, N., Casavecchia, P., Rosi, M., Skouteris, D., Berteloite, C., Le Picard, S. D., Canosa, A., \& Sims, I. R. 2009, J. Phys. Chem. A, in press, DOI: $10.1021 / j p 906299 \mathrm{v}$

Guo, Y., Gu, X., Zhang, F., Sun, B. J., Tsai, M. F., Chang, A. H. H., \& Kaiser, R. I. 2007, J. Phys. Chem. A 111, 3241. 